\title{
Analysis of Genes Related to Angiotensin II-Induced Arterial Injury Using a Time Series Microarray
}

\author{
Yun-Long Zhang ${ }^{\mathrm{a}} \quad$ Lian-Yun Zhi $^{\mathrm{b}} \quad$ Lei-Xin Zou $^{\mathrm{a}}$ Chen Chen $^{\mathrm{a}} \quad$ Jie Bai ${ }^{\mathrm{a}}$ \\ Qiu-Yue Lin ${ }^{b}$ Song Lai ${ }^{b}$ Lei Wang ${ }^{b}$ Ying Liub Hui-Hua Lia,b
}

aSchool of Public Health, Dalian Medical University, Dalian, bDepartment of Cardiology, Institute of Cardiovascular Diseases, First Affiliated Hospital of Dalian Medical University, Dalian, China

\section{Key Words}

Angiotensin II • Hypertension • Artery remodeling • Microarray • Prkaca

\begin{abstract}
Background/Aims: Angiotensin II (Ang II)-mediated hypertension is a major risk factor for cardiovascular diseases. Ang II induces changes in vessel structure and function through the activation of genes related to signaling pathways. However, the changes in the gene expression profiles of blood vessels in response to Ang II remain unclear. Methods: Wild-type C57BL/6 mice were infused with Ang II (1500 $\mathrm{ng} / \mathrm{kg} / \mathrm{min})$ using an osmotic pump for 1,3 , and 7 days. Vascular wall inflammation and remodeling were evaluated by pathological examination. Timeseries microarray and quantitative PCR analyses were performed. Bioinformatics analyses were conducted to identify key genes, pathways, and biological processes. Results: After Ang II infusion, blood pressure and aortic remodeling were increased over time. Microarray analysis identified a totally of 3631 differentially expressed genes in aortas at days 1, 3, and 7 of Ang II infusion. These genes were involved in multiple biological processes, including cell adhesion, angiogenesis, cell migration, protein phosphorylation, immune system, and cell cycle, which may play important roles in regulating Ang II-induced arterial injury during hypertension. The genes were classified into 50 profiles by hierarchical cluster analysis, and finally, 14 significant profiles were identified. Among these genes, protein kinase CAMP-activated catalytic subunit alpha (Prkaca), a gene that directly regulated 137 neighboring genes, was located at the center of the gene network in Ang II-infused aortas. Further, Prkaca protein expression and cAMP level were downregulated in a time-dependent manner in Ang II-infused aortas. Conclusions: The combined use of DNA microarrays and cluster and gene network analyses identified Prkaca as a key Ang II-responsive gene that may mediate early vascular injury and hypertension.
\end{abstract}

(C) 2018 The Author(s)

Published by S. Karger AG, Basel

Y.-L. Zhang and L.-Y. Zhi contributed equally to this work.

\begin{tabular}{ll}
\hline Ying Liu, MD & Department of Cardiology, Institute of Cardiovascular Diseases \\
and Hui-Hua Li, MD, PhD & First Affiliated Hospital of Dalian Medical University, Dalian 116011 (China) \\
& E-Mail yingliu.med@gmail.com, hhli1935@aliyun.com
\end{tabular}




\section{Cellular Physiology Cell Physiol Biochem 2018;48:983-992

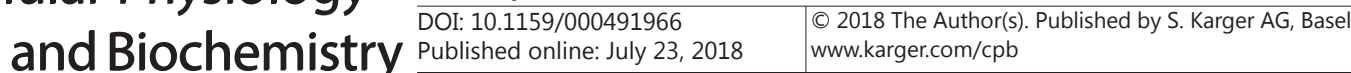

Zhang et al.: Genes Related to Ang II-Induced Arterial Remodeling

\section{Introduction}

Hypertension is a major risk factor for various cardiovascular diseases, including stroke, and heart failure, and renal diseases. It is generally characterized by arterial remodeling, such as thickening of the media and intima and reduced lumen diameter [1]. Substantial evidence suggests that the renin-angiotensin system (RAS) plays a central role in blood pressure regulation and vascular remodeling [2]. Angiotensin II (Ang II) is a powerful vasoconstrictor of blood vessels. Chronic elevation of Ang II may result in hypertension and vascular remodeling, whereas repression of Ang II or inhibitio of its action markedly improves hypertension and heart diseases [3]. However, little is known about the changes in gene expression in the arteries during the early stage of Ang II infusion.

Vascular remodeling is a hallmark of most vascular disorders including hypertension, atherosclerosis, post-angioplasty restenosis, allograft vasculopathy, and pulmonary disease [4]. Many studies indicate that Ang II mediates inflammation, vascular growth, remodeling and oxidative stress through multiple mechanisms, including disruption of the endothelial nitric oxide synthase (eNOS) pathway, decrease of nitric oxide (NO) activity, increase of oxidative stress, matrix production and remodeling and function, the precise dysfunction of the endothelium [5]. Although Ang II plays a critical role in the regulation of arterial molecular mechanism by which Ang II causes early arterial injury remains unknown. Recently, mRNA microarray assays have been applied to determine alterations of gene expression profiles in various tissues, which has identified some critical genes for inherited cardiovascular diseases [6, 7].

In this study, we aimed to analyze the gene expression profiles of arteries in an Ang IIinduced hypertension mouse model using time-series microarrays. Our data revealed that a total of 3631 genes were significantly altered in arteries at days 1, 3, and 7 of Ang II infusion. These genes were classified into 50 clusters based on their expression pattern. Of these, 14 profiles that contained 2894 genes were statistically significant. Importantly, Protein kinase cAMP-activated catalytic subunit alpha (Prkaca), also known as PKAC-alpha, was localized at the center of the gene network and may play a key role in Ang II-induced arterial injury and hypertension.

\section{Materials and Methods}

\section{Animal models}

C57BL/6J male mice (8-10 weeks-old) were infused with Ang II (1500 ng/kg/min) using an osmotic pump (Model 1007D; Alzet, Cupertino, CA) for 1, 3, and 7 days. Systolic blood pressure was detected by the tail-cuff method before starting treatment and every day after Ang II infusion, as described previously [8, 9]. The investigation conformed to the Guide for the Care and Use of Laboratory Animals published by the U.S. National Institutes of Health. The procedures were approved by and performed in accordance with the Animal Care and Use Committee of Dalian Medical University.

\section{Histological examination}

At days 1, 3, and 7 after Ang II infusion, the aortas were fixed in 4\% phosphate-buffered paraformaldehyde. Arterial sections $(5 \mu \mathrm{m})$ were stained with hematoxylin and eosin (H\&E), Masson, and immunohistochemistry as described previously [8]. The images were evaluated in a blinded fashion by a pathologist and were captured using a Nikon Labophot 2 microscope (Nikon, Tokyo, Japan).

\section{Microarray assay and comprehensive bioinformatics analysis}

The mice were euthanized by an overdose of pentobarbital (100 mg/kg, intraperitoneal injection) at days 1, 3, and 7 of Ang II infusion. Arteries ( $n=3$ per group) were removed and frozen in liquid nitrogen. Total RNA was isolated with TRIzol (Invitrogen, Carlsbad, CA) from aortas at each time point according to the manufacturer's instructions $[8,9]$. Gene expression profiling was performed using the mouse Genome 4302.0 array according to the manufacturer's instructions (Affymetrix, Inc., Santa Clara, CA) [9-11]. Fifteen 


\section{Cellular Physiology Cell Physiol Biochem 2018;48:983-992 \begin{tabular}{l|l|l} 
and Biochemistry $10.1159 / 000491966$ & $\begin{array}{l}\text { D } 2018 \text { The Author(s). Published by S. Karger AG, Basel } \\
\text { Pww.karger.com/cpb }\end{array}$
\end{tabular} Zhang et al.: Genes Related to Ang II-Induced Arterial Remodeling}

micrograms of biotin-labeled complementary RNA was fractionated and hybridized to the Affymetrix GeneChip. Microarray data were analyzed as described previously [9-11]. The gene expression data are available at the Gene Expression Omnibus website under the website under accession number GSE105114 (http:// www.ncbi.nlm.nih.gov/geo/).

The intensity of gene expression was geometrically averaged and expressed as the fold ratio of Ang II-infused mice compared with the saline group for each time point. Comprehensive bioinformatics analysis was used to enrich the dataset for genes that were associated with Ang II-induced arterial injury and hypertension, including analysis of differential genes, series test of clusters, gene ontology (GO), pathways, and gene co-expression networks, as described in our recent publication [9].

Verification of microarray results by quantitative real-time PCR analysis

The differentially expressed genes that were observed in the microarray assay were verified by qPCR ( $\mathrm{n}$ = 4-6 per group), which was performed with an Applied Biosystems 7500 Fast Real-Time PCR System (Applied Biosystems, Foster City, CA). The cycling conditions consisted of an initial, single cycle of $4 \mathrm{~min}$ at $95^{\circ} \mathrm{C}$, followed by 40 cycles of $10 \mathrm{~s}$ at $95^{\circ} \mathrm{C}, 45 \mathrm{~s}$ at $60^{\circ} \mathrm{C}$, and fluorescence acquisition, then $95^{\circ} \mathrm{C}$ for $1 \mathrm{~min}$, and $60^{\circ} \mathrm{C}$ for $15 \mathrm{~s}$; a melting curve was carried out from $60^{\circ} \mathrm{C}$ to $95^{\circ} \mathrm{C}$, and fluorescence was collected every $0.5^{\circ} \mathrm{C}$. The level of gene expression was quantified relative to the expression of GAPDH. The primers used are listed in Table 1.

\section{Immunoblotting analysis}

Immunoblotting analysis was performed as described previously [12, 13]. Aortas were lysed with lysis buffer (20 mM Tris [pH 7.5], 1 mM EDTA, 150 mM NaCl, 1 mM EGTA, 1 mM $\beta$-glycerophosphate, 1\% Triton X-100, $2.5 \mathrm{mM}$ sodium pyrophosphate, $1 \mathrm{mM} \mathrm{Na}_{3} \mathrm{VO}_{4}, 4 \mu \mathrm{g} / \mathrm{mL}$ aprotinin, $4 \mu \mathrm{g} / \mathrm{mL}$ leupeptin, $4 \mu \mathrm{g} / \mathrm{ml}$ pepstatin, and $1 \mathrm{mM}$ PMSF).Proteins ( $50 \mu \mathrm{g}$ ) from each sample were separated by sodium dodecyl sulfate polyacrylamide gel electrophoresis, and transferred to polyvinylidene difluoride membranes, and probed with a primary antibody against Prkaca (1:800), and detected with a horseradish peroxidase-conjugated secondary antibody.

\section{Assay for cAMP and cGMP activity}

Commercial kits were used to assess cAMP and cGMP levels of arterial tissues according to the manufacturer's instructions (BioVision, Milpitas, CA) [14].

\section{Statistical analysis}

Data are presented as the mean \pm standard error of the mean (SEM). Differences between groups were analyzed by non-parametric tests (Kruskal-Wallis or Mann-Whitney) or by the parametric test oneway analysis of variance followed by the Tukey-Kramer test for group differences. $P<0.05$ was considered statistically significant.

\section{Results}

Ang II infusion induces hypertension and aortic injury in mice

To determine the global alterations of gene expression and identify genes that are important to Ang II-induced arterial injury, a mouse model of hypertension was successfully 
induced by Ang II infusion. Ang II infusion increased systolic blood pressure in a timedependent manner (Fig. 1A). The thickness of the arterial wall was also significantly increased in Ang II-infused aortas (Fig. 1B). Moreover, continuous infusion of Ang II caused aortic infiltration of Mac-2-positive macrophages and collagen deposition at days 3 and 7 (Fig. 1C, 1D). In addition, Ang II-induced aortic fibrosis was further supported by the increased expression of Col1a1, Col1a2, Col3a1 and Col5a1 mRNA, as observed using the microarray (Fig. 1E).

\section{Genes detected by microarray assay in mouse aorta after Ang II infusion}

To characterize the gene expression profiles of arteries that were associated with the Ang II response, time-series microarrays ( $\mathrm{n}=3$ per time point) were performed with artery samples at days 1, 3, and 7 of Ang II infusion (Fig. 2A). We observed a total of 3631 genes that had a $P$-value and false discovery rate (FDR) $<0.05$ in Ang II-infused arteries in at least one time point as compared with saline-treated control. Among them, 799, 1853, and 3483 genes were markedly altered at days 1,3 , and 7 , respectively.

\section{Analysis of GO terms and pathways}

After Ang II infusion, we observed the most significantly overrepresented GO terms $(P<0.001)$, including cell adhesion, angiogenesis, cell migration, protein phosphorylation, immune system process, cell cycle, response to hypoxia, and mitotic nuclear division. (Fig. 2B). Here we found that a total of 112 pathways were significantly altered in Ang II-treated arteries compared with saline control, which were associated with focal adhesion, chemokine signaling pathway, PI3K-Akt signaling pathway, cell cycle, regulation of actin cytoskeleton, vascular smooth muscle contraction, tumor necrosis factor (TNF) signaling pathway, nuclear factor-k-gene binding (NF-kB) signaling pathway, B cell receptor signaling pathway, extracellular matrix (ECM)-receptor interaction, transforming growth factor (TGF)- $\beta$

Fig. 1. Systolic blood pressure and arterial pathological examination in Ang II-induced mouse model. (A) Wild-type mice were infused with Ang II (1500 ng/kg/min) for 1,3 , and 7 days. Systolic blood pressure was measured by the tail cuff method ( $n=6$ per group). (B) H\&E staining of arterial sections (left). Quantification of aortic wall thickness $(\mathrm{n}=3$, right). (C) Immunohistochemical staining of arterial sections (left). Quantification of Mac-2 positive macrophage area (right, $\mathrm{n}=3$ ). (D) Masson's trichrome staining of arterial sections (left). Quantification of aortic fibrotic area (right, $n=3$ ). (E) Microarray data of the mRNA expression of Col1a1, Col1a2, Col3a1 and Col5a1 ( $n=3$ per group). Data are shown as mean \pm SEM. ${ }^{*} \mathrm{P}<0.05$ versus control.

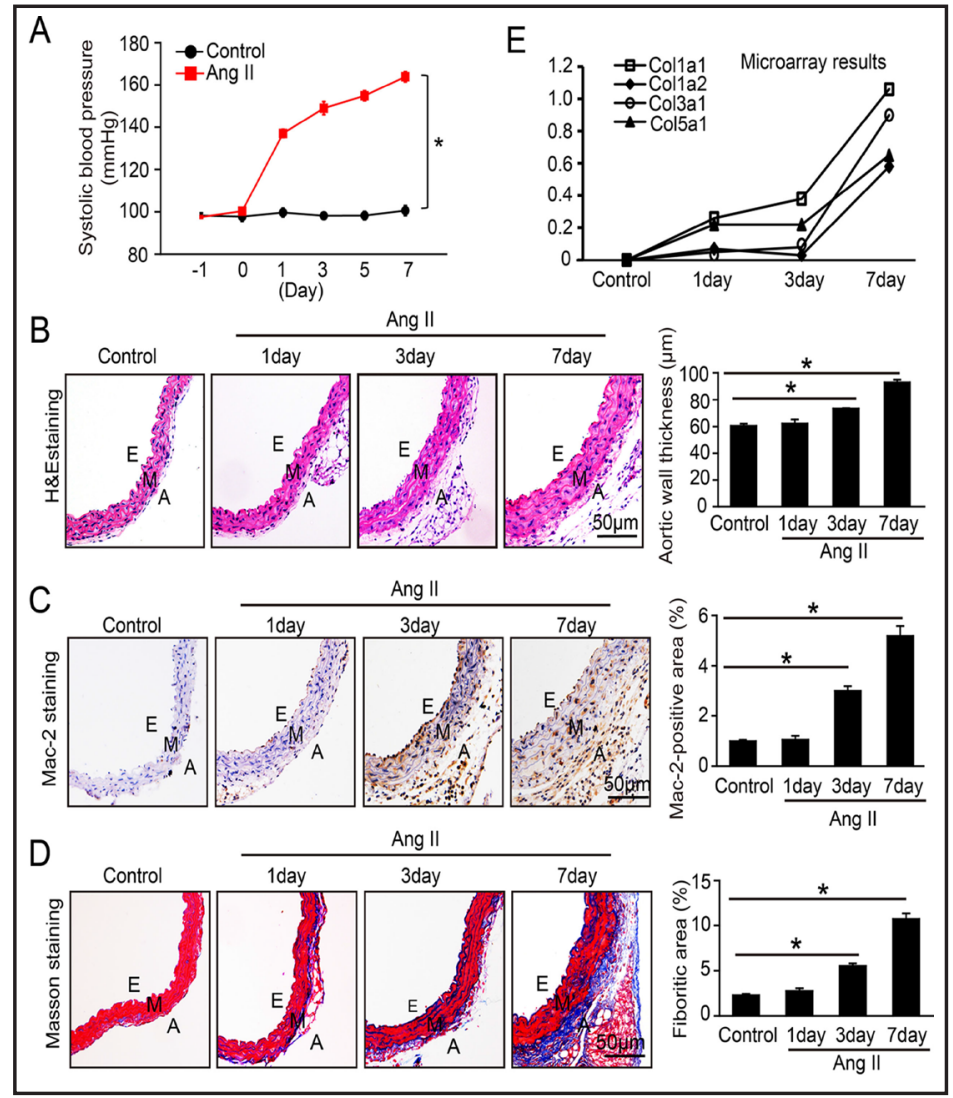


Fig. 2. Analysis of GO terms and KEGG pathways. (A) The heat map illustrates the expression differences of all mRNAs on the microarray between Ang IIinfused arteries and control at the 3 different time points $(1,3$, and 7 days) $(n=3)$. The map contains LgP intensity/fold change ratios that are color coded with red

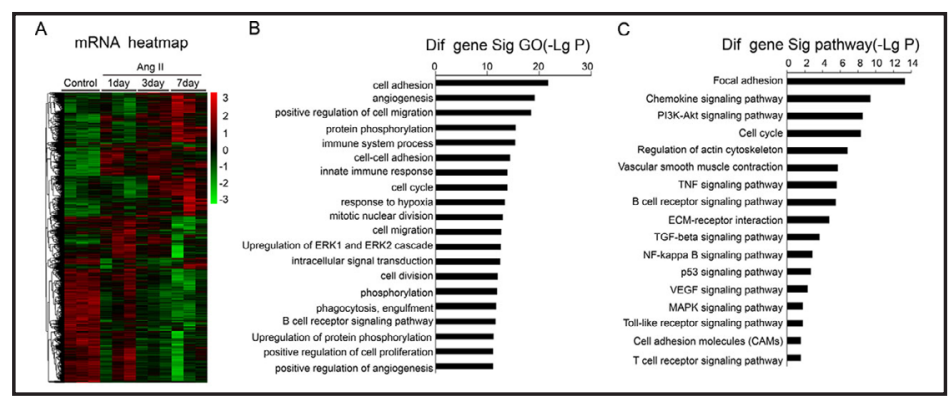
corresponding to up-regulation and Green to down-regulation. (B) GO category analysis based on biological process for differentially expressed genes. (B) KEGG pathway analysis for the differentially expressed genes in the arteries after Ang II infusion. LgP is the logarithm of P-value.

Fig. 3. The clustering analysis of significant differential genes in arteries after Ang II infusion. The differentially expressed genes were classified into 50 profiles by hierarchical cluster analysis. Each box represents a model expression profile. Fourteen expression patterns (No. 9, 42, 1, 40, 34, 48, $29,47,18,23,49,0,12$, and 11) of genes showed statistically significant $\mathrm{P}$-values $(\mathrm{P}<0.00001)$ (colored boxes). The number in the box represents the ID of the profile and P-value.

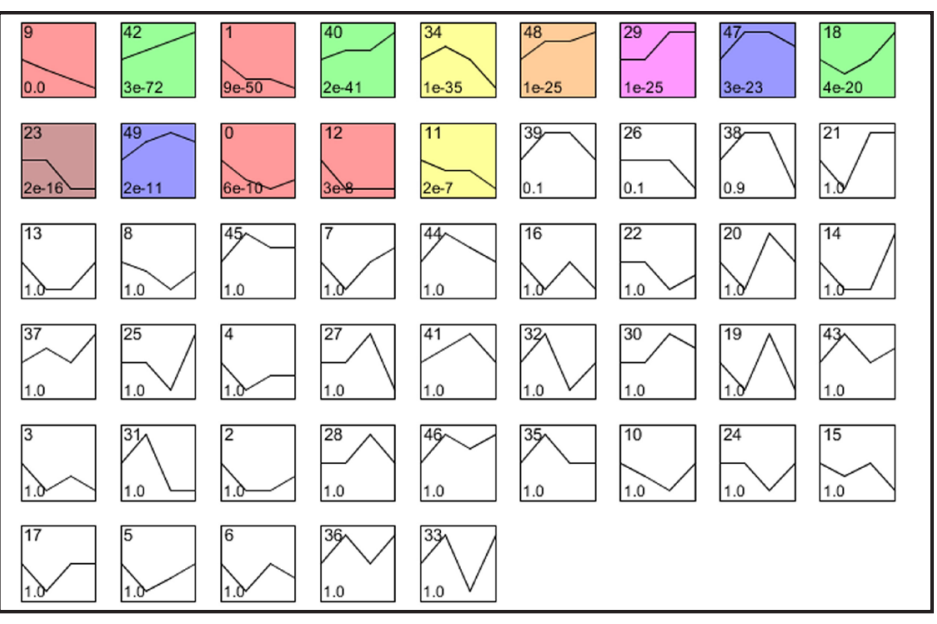

signaling pathway, etc. (Fig. 2C). Several of the observed pathways have been implicated in Ang II-induced hypertension, fibrosis, and inflammation.

\section{Cluster analysis}

To profile the gene expression time series and identify the most probable set of clusters generating the observed time series, 3631 genes that were differentially expressed in arteries were classified into 50 profiles by hierarchical cluster analysis after Ang II infusion (Fig. 3). Of these clusters, 14 significant profiles were identified, namely, No. 9, 42, 1, 40, 34, $48,29,47,18,23,49,0,12$, and 11, which contained a total of 2894 genes (Fig. 3, 4). The expression of genes in profiles No. 42, 40, and 48 was constantly increased at days 1,3 , and 7. The expression of genes in profile No. 29 was increased at days 1 and 3 , and progressively increased to a high level at day 7. The expression of genes in profile No. 34 was increased at day 1 , and then gradually decreased at days 3 and 7 . The expression of genes in profiles No. 47 and 49 was increased at days 1 and 3 , and then gradually decreased at day 7 . The expression of genes in profile No. 18 was decreased at day 1 , and then gradually increased at days 3 and 7. The expression of genes in profiles No. 9, 1,23, 0,12 , and 11 was decreased significantly at days 1,3 , and 7. Interestingly, profile No. 9, the most significant profile $(P=0.000)$ contained 724 genes that were the most abundant and exhibited decreased expression at all times during Ang II infusion (Fig. 3, 4).

\section{Verification of gene expression by qPCR analysis}

To confirm the results from the microarray analysis, qPCR was performed on the same arteries. The relative expression levels of 13 differentially expressed genes from 9 
significant profiles (Fig. 4), namely No. 9 (Prkaca), No. 42 (Gngt2 and Gngt1), No.1 (Prkacb and Pik3ca), No. 40 (Arrb2 and Gng2), No. 34 (Kras), No. 48 (Pik3r5), No. 29 (Ccr7 and Cyp2c54), No. 47 (Csf1r), and No. 18 (Cyp2c66), were analyzed. As shown in Fig. 6, Prkaca, Prkacb and Pik3ca were decreased at all time points; Cyp2c66 was decreased at day 1, increased at day 3 , and continued to increase at day 7; Csf1r was progressively increased at day 1 and 3 but decreased at day 7; Kras was initially increased at day 1 , but its expression decreased gradually at days 3 and 7; Ccr7 and Cyp2c54 were increased gradually at days 1 and 3 , and then remained at a high level at day 7; and Gngt2, Gngt1, Arrb2, Gng2 and Pik3r5 were constantly increased at days 1,3 , and 7; All of the qPCR findings were consistent with those of the microarray analysis (Fig. 5).

\section{Gene co-expression network analysis \\ To identify genes} that have central roles in hypertension and arterial remodeling induced by Ang II infusion, based on the degree, K-core value, and betweenness centrality, 294 candidate genes selected from the 14 significant profiles, namely No. $9,42,1$, $40,34,48,29,47,18,23,49$, 0,12 , and 11 , were further analyzed using a gene coexpression network with a k-core algorithm (Fig. 6). Degree is defined as the number of links one node has to other nodes. Genes

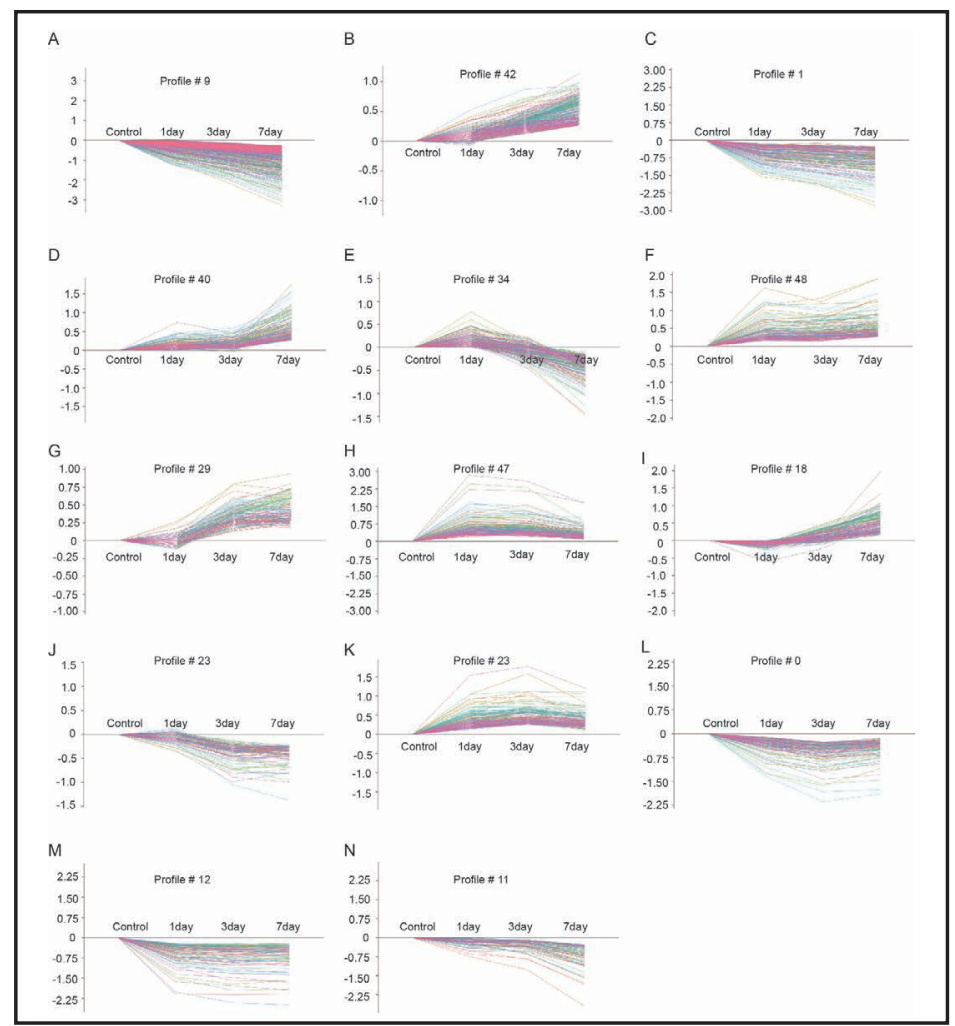

Fig. 4. The time-series patterns of gene expression profiles. No. 9 (A), 42 (B), 1 (C), 40 (D), 34 (E), 48 (F), 29 (G), 47 (H),18 (I), 23 (J), 49 (K), $0(\mathrm{~L}), 12(\mathrm{M})$ and $11(\mathrm{~N})$ detailed in the artery after Ang II infusion (n =3). The horizontal axis represents time points, and the vertical axis shows the time series of gene expression levels for the gene after Log normalized transformation.

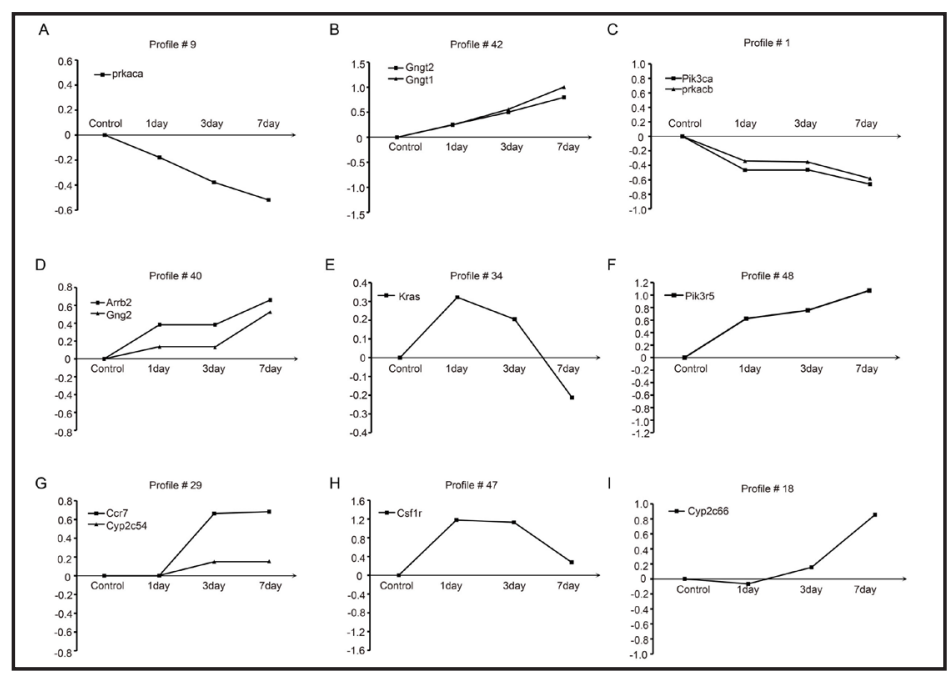

Fig. 5. Verification of microarray results by qPCR analysis in the artery after Ang II infusion. The expression levels of 13 differentially genes from both significant and non-significant profiles were confirmed by qPCR analysis, including Prkaca, Gngt2, Gngt1, Pik3ca, Prkacb, Arrb2, Gng2, Kras, Pik3r5, Ccr7, Cyp2c54, Csfr1 and Cyp2c66. GAPDH as an internal control. Data were presented as mean \pm SEM $(n=4-6$ per group). 
Fig. 6. Gene co-expression network. 294 most important genes selected from 14 significant profiles, including No. 9, 42, 1 , $40,34,48,29,47,18,23,49,0$, 12 and 11 were further analyzed by gene co-expression network with k-core algorithm. Cycle nodes represent genes, and edges between two nodes represent interactions between genes which were quantified by degree. Genes with higher degrees are more centralized in the network and have a stronger capacity of regulating adjacent genes.

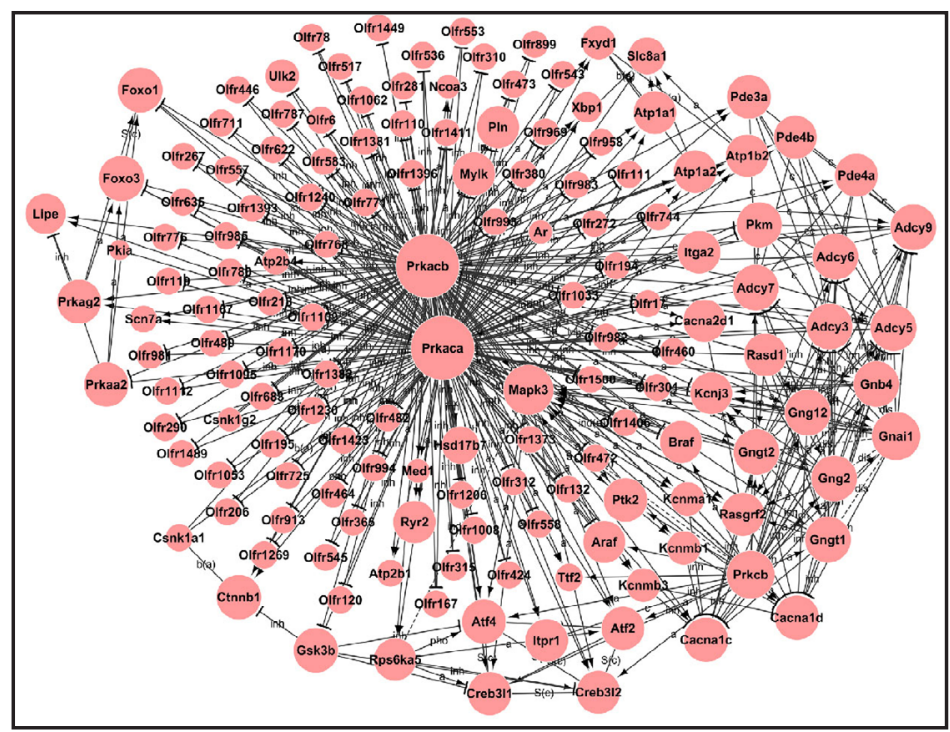

with higher degrees occupied more central positions in the network and had a stronger capacity to modulate adjacent genes. Among these genes, Prkaca had the highest degree, appeared at the center of the gene network, and directly regulated 137 neighboring genes (Fig. 6). The betweenness centrality of Prkaca was also higher (0.055540334) than that of the other genes (Table 2). Overall, the results indicate that Prkaca may play a central role in the regulation of hypertension and arterial remodeling (Fig. 6).

Validation of Prkaca expression by immunoblotting analysis

We next assessed the effect of Ang II infusion on Prkaca expression. Immunoblotting analysis showed that the protein level of Prkaca was significantly decreased in Ang II-infused aortas (Fig. 7A). Since PKA can be activated by cAMP, we tested whether Ang II infusion affected cAMP levels. We found that cAMP but not cGMP was also reduced in Ang II-infused aortas (Fig. 7B, 7C).

\section{Discussion}

In this study, we used timeseries microarray and comprehensive bioinformatics analyses to identify differentially expressed genes, GO terms, and pathways. We identified a totally of 3631 differentially expressed genes in Ang II-infused arteries that were involved in multiple biological processes, including cell adhesion, protein
Table 2. 26 genes identified by gene co-expression network with k-core algorithm

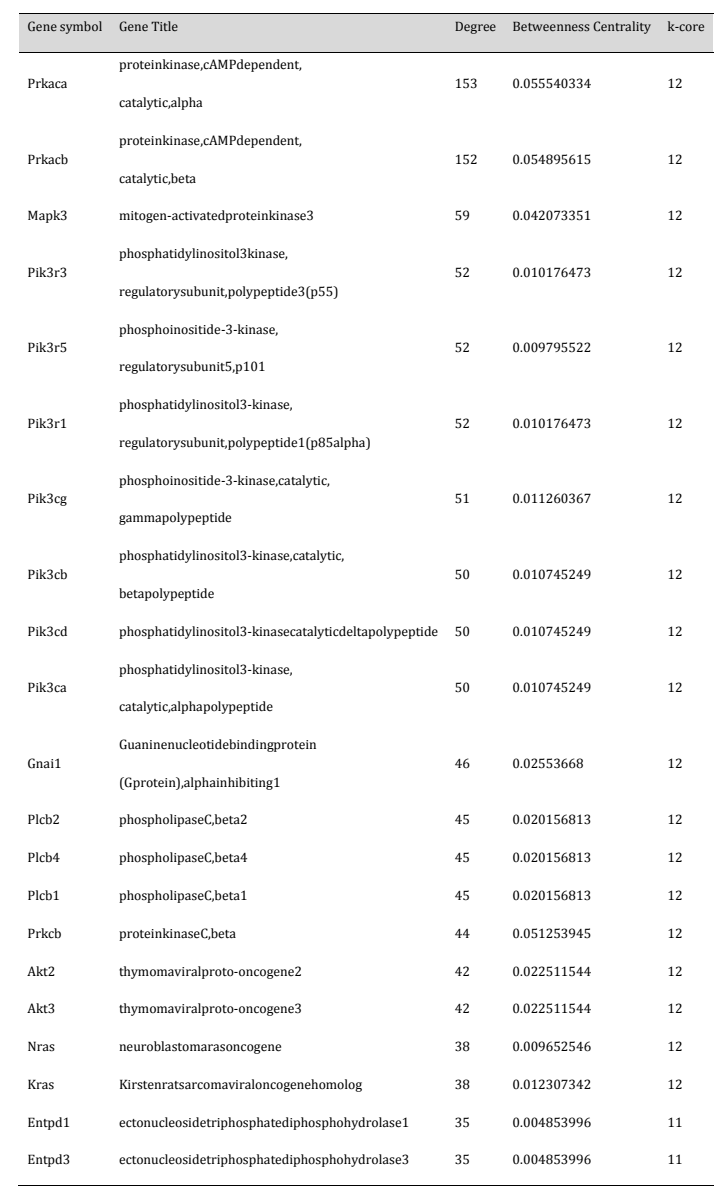


phosphorylation, immune system, and cell cycle. Further, we classified these genes into 50 profiles by hierarchical cluster analysis, and identified 14 significant profiles. Gene coexpression network analysis demonstrated that the Prkaca was localized in the center of the gene co-expression network. We also confirmed that Prkaca expression was significantly decreased in Ang II-infused arteries. These results suggest that Prkaca may be involved in regulating Ang IIinduced vascular injury and hypertension.

Pathological vascular remodeling is a major feature of vascular diseases and involves changes in the function of endothelial cells, vascular smooth muscle cell (SMC) proliferation and migration, apoptosis, inflammation and ECM accumulation [2]. Ang II causes SMC growth and migration and stimulates the expression of vascular remodeling-related genes. Ang II activates a number of different intracellular signal transduction pathways via the AT1 receptor, resulting in the activation of epidermal growth factor and platelet-derived growth factor receptors, which are responsible for the induction of vascular remodeling, indicating that Ang II plays an important role in this process [5]. Thus, the identification of molecules involved in vessel remodeling could promote the development of improved treatments for vascular diseases and hypertension.

Microarray analysis has been used widely to examine the changes of gene expression profiles, and to identify genes that are involved in Ang II-induced response in arterial cells [15, 16]. Ang II infusion significantly altered multiple pathways, including focal adhesion, chemokine signaling pathway, PI3K-Akt signaling pathway,

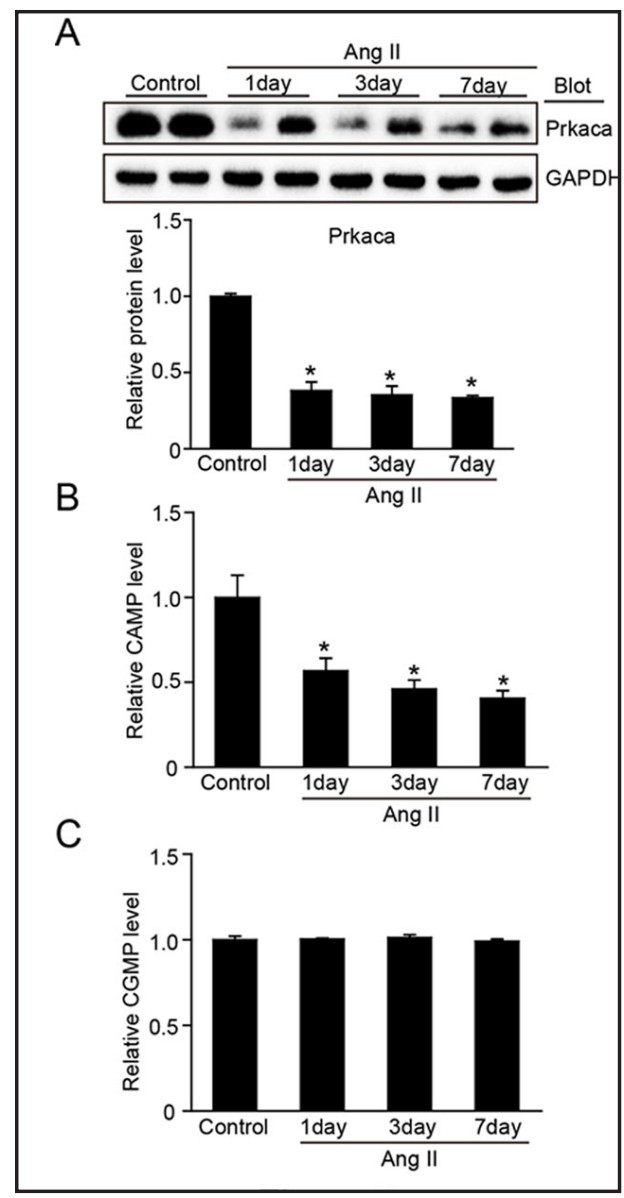

Fig. 7. Validation of Prkaca protein expression. (A) Immunoblotting analysis of Prkaca protein expression in the artery after Ang II infusion. GAPDH as an internal control. (B, C) Measurement of cAMP and CGMP levels in the artery after Ang II infusion. Data were presented as mean \pm SEM $(n=4$ per group). ${ }^{*} \mathrm{P}<0.05$ versus control. cell cycle, vascular smooth muscle contraction, B cell receptor signaling pathway, ECM-receptor interactions, TGF- $\beta, N F-k B, p 53$, and vascular endothelial growth factor (VEGF) signaling pathways. (Fig. 2C), which are the main mechanisms involved in Ang II-induced inflammation, fibrosis, and angiogenesis [17-19]. Therefore, these data provide novel insights into the molecular events of Ang II-mediated arterial remodeling and hypertension.

It is well known that Ang II plays a central role in the regulation of blood pressure and vascular injury through AT1R-mediated inflammation, oxidative stress and vascular remodeling [5], but the key genes that are involved in these pathophysiological processes remain unclear. Here, our cluster and gene co-expression network analyses showed that Prkaca appears at the core of the gene network (Fig. 5, Table 2). PKA is a tetrameric protein, consisting of two regulatory (R) dimers and two catalytic (C) subunits. The addition of cAMP causes a conformational change in the anchored $R$ subunit that releases the $C$ subunit, which is then free to interact with and phosphorylate downstream targets [20,21]. The PKA catalytic subunits $C \alpha$ and $C \beta$ are encoded by Prkaca and Prkacb, respectively [22]. Increasing evidence demonstrates that PKA regulates a number of different physiological reactions in response to cAMP, including cell division, insulin resistance, metabolism, and cardiac function [22]. 
Several studies also showed that hypertrophic stimuli, such as Ang II and phenylephrine, can increased intracellular cAMP levels and subsequently activate PKA, which phosphorylates cAMP response element binding protein, leading to cardiac hypertrophy [23, 24]. Moreover, the receptor tyrosine kinases EGFR and PDGFR can phosphorylate Prkaca at Tyr-331, resulting in an increase of its catalytic efficiency [25]. Further, Prkaca also phosphorylates a number of substrates, including HSF1, NFKB1, PSMC5/RPT6, PJA2, RYR2, RORA, and VASP, which activate multiple signaling pathways. The cardiac-specific overexpression of Prkaca results in cardiomyopathy and sudden death [20]. However, it is unknown whether Prkaca is regulated by Ang II in the arteries. Here, our microarray results showed that Ang II infusion markedly decreased Prkaca expression at mRNA level and protein level (Fig. 7A). Accordingly, cAMP but not cGMP was also reduced in Ang II-infused arteries (Fig. 7B, 7C). Moreover, Prkaca directly regulated 137 neighboring genes (Fig. 6), suggesting that Prkaca may play a critical role in Ang II-infused vascular diseases and hypertension.

In conclusion, our data showed that 3631 genes were differently expressed in Ang IItreated aortas compared with control at different time points. Integrative analysis indicated that Prkaca may play a central role in Ang II-induced aortic remodeling and hypertension. Further studies should be undertaken to determine the mechanism by which Ang II downregulates the expression of Prkaca in the aorta; and to uncover the role of Prkaca in regulating Ang II-induced hypertension and vascular injury.

\section{Acknowledgements}

This work was supported by grants from the State Key Program of National Natural Science Foundation of China (81630009 to Dr. Li), the Youth Science Fund Program of National Natural Science Foundation of China (81400247 to Dr. Liu), and the Chang Jiang Scholar Program of China (T2011160 to Dr. Li).

\section{Disclosure Statement}

No conflict of interests exists.

\section{References}

1 Oparil S, Zaman MA, Calhoun DA: Pathogenesis of hypertension. Ann Intern Med 2003;139:761-776.

-2 Renna NF, de Las Heras N, Miatello RM: Pathophysiology of vascular remodeling in hypertension. Int J Hypertens 2013;2013:808353.

3 Clarke C, Flores-Munoz M, McKinney CA, Milligan G, Nicklin SA: Regulation of cardiovascular remodeling by the counter-regulatory axis of the renin-angiotensin system. Future Cardiol 2013;9:23-38.

4 Gibbons GH, Dzau VJ: The emerging concept of vascular remodeling. N Engl J Med 1994;330:1431-1438.

5 Mehta PK, Griendling KK: Angiotensin II cell signaling: physiological and pathological effects in the cardiovascular system. Am J Physiol Cell Physiol 2007;292:C82-97.

-6 Wu HJ, Zhang CY, Zhang S, Chang M, Wang HY: Microarray Expression Profile of Circular RNAs in Heart Tissue of Mice with Myocardial Infarction-Induced Heart Failure. Cell Physiol Biochem 2016;39:205-216.

7 Li TT, Li XY, Jia LX, Zhang J, Zhang WM, Li YL, Qi YF, Du J: Whole Transcriptome Analysis of Hypertension Induced Cardiac Injury Using Deep Sequencing. Cell Physiol Biochem 2016;38:670-682.

8 Wang L, Zhao XC, Cui W, Ma YQ, Ren HL, Zhou X, Fassett J, Yang YZ, Chen Y, Xia YL, Du J, Li HH: Genetic and Pharmacologic Inhibition of the Chemokine Receptor CXCR2 Prevents Experimental Hypertension and Vascular Dysfunction. Circulation 2016;134:1353-1368.

-9 Zhang JS, Zhang YL, Wang HX, Xia YL, Wang L, Jiang YN, Li HH, Liu Y: Identification of genes related to the early stage of Angiotensin II-induced acute renal injury by microarray and integrated gene network analysis. Cell Physiol Biochem 2014;34:1137-1151. 


\section{Cellular Physiology Cell Physiol Biochem 2018;48:983-992 \begin{tabular}{l|l} 
DOI: 10.1159/000491966 & $\begin{array}{l}\text { O 2018 The Author(s). Published by S. Karger AG, Basel } \\
\text { www.karger.com/cpb }\end{array}$
\end{tabular} \\ Zhang et al.: Genes Related to Ang II-Induced Arterial Remodeling}

10 Dang MQ Zhao XC, Lai S, Wang X, Wang L, Zhang YL, Liu Y, Yu XH, Liu Y, Li HH, Xia YL: Gene expression profile in the early stage of angiotensin II-induced cardiac remodeling: a time series microarray study in a mouse model. Cell Physiol Biochem 2015;35:467-476.

11 Yang D, Zeng Y, Tian C, Liu J, Guo SB, Zheng YH, Li HH: Transcriptomic analysis of mild hypothermiadependent alterations during endothelial reperfusion injury. Cell Physiol Biochem 2010;25:605-614.

12 Qin XY, Zhang YL, Chi YF, Yan B, Zeng XJ, Li HH, Liu Y: Angiotensin II Regulates Th1 T Cell Differentiation Through Angiotensin II Type 1 Receptor-PKA-Mediated Activation of Proteasome. Cell Physiol Biochem 2018;45:1366-1376.

-13 Wang L, Li YL, Zhang CC, Cui W, Wang X, Xia Y, Du J, Li HH: Inhibition of Toll-like receptor 2 reduces cardiac fibrosis by attenuating macrophage-mediated inflammation. Cardiovasc Res 2014;101:383-392.

14 Zhang B, Sun N, Mu X, Zhi L, Zhai L, Jiang Y, Fu Z, Yao Z: G Protein Alpha S Subunit Promotes Cell Proliferation of Renal Cell Carcinoma with Involvement of Protein Kinase A Signaling. DNA Cell Biol 2017;36:237-242.

15 Jaffe IZ, Mendelsohn ME: Angiotensin II and aldosterone regulate gene transcription via functional mineralocortocoid receptors in human coronary artery smooth muscle cells. Circ Res 2005;96:643-650.

16 Madhur MS, Lob HE, McCann LA, Iwakura Y, Blinder Y, Guzik TJ, Harrison DG: Interleukin 17 promotes angiotensin II-induced hypertension and vascular dysfunction. Hypertension 2010;55:500-507.

17 Hunyady L, Catt KJ: Pleiotropic AT1 receptor signaling pathways mediating physiological and pathogenic actions of angiotensin II. Mol Endocrinol 2006;20:953-970.

-18 Huang Y, Noble NA, Zhang J, Xu C, Border WA: Renin-stimulated TGF-beta1 expression is regulated by a mitogen-activated protein kinase in mesangial cells. Kidney Int 2007;72:45-52.

$>19$ Neilson EG: Mechanisms of disease: Fibroblasts--a new look at an old problem. Nat Clin Pract Nephrol 2006;2:101-108.

-20 Antos CL, Frey N, Marx SO, Reiken S, Gaburjakova M, Richardson JA, Marks AR, Olson EN: Dilated cardiomyopathy and sudden death resulting from constitutive activation of protein kinase a. Circ Res 2001;89:997-1004.

-21 Brandon EP, Idzerda RL, McKnight GS: PKA isoforms, neural pathways, and behaviour: making the connection. Curr Opin Neurobiol 1997;7:397-403.

-22 Lohse MJ, Engelhardt S: Protein kinase a transgenes: the many faces of cAMP. Circ Res 2001;89:938-940.

23 Chen H, Wang X, Tong M, Wu D, Wu S, Chen J, Wang X, Wang X, Kang Y, Tang H, Tang C, Jiang W: Intermedin suppresses pressure overload cardiac hypertrophy through activation of autophagy. PLoS One 2013;8:e64757.

24 Markou T, Hadzopoulou-Cladaras M, Lazou A: Phenylephrine induces activation of CREB in adult rat cardiac myocytes through MSK1 and PKA signaling pathways. J Mol Cell Cardiol 2004;37:1001-1011.

25 Caldwell GB, Howe AK, Nickl CK, Dostmann WR, Ballif BA, Deming PB: Direct modulation of the protein kinase A catalytic subunit alpha by growth factor receptor tyrosine kinases. J Cell Biochem 2012;113:3948. 